\title{
ESTUDIO DE FORMAS DE SOLUCIÓN DE SITUACIONES PROBLEMA DESDE LAS MATEMÁTICAS ESCOLARES Y LAS MATEMÁTICAS EXTRAESCOLARES
} WAYS TO SOLVE PROBLEM SITUATIONS FROM SCHOOL MATHEMATICS AND EXTRACURRICULAR MATHEMATICS

\author{
Hilbert Blanco Álvarez ${ }^{1}$ \\ Pedro Yilber Nazate Calpa² \\ Universidad de Nariño \\ San Juan de Pasto, Nariño, Colombia
}

\section{RESUMEN}

Este estudio tiene como objetivo describir formas de solución para situaciones problema, desde la matemática escolar y la matemática extraescolar. La problemática se enmarca en el campo investigativo de la Etnomatemática, luego de realizar una revisión de investigaciones en ámbitos nacionales e internacionales que aluden a tensiones entre las matemáticas académicas y extraescolares. Se hizo uso de una metodología cualitativa e interpretativa que contempló dos 1 Doctor en Educación por la Universidad de Granada, España. Docente departamento de matemáticas y estadística, Universidad de Nariño, Pasto, Nariño, Colombia. Correo: hilbla@udenar.edu.co. Orcid: https://orcid. org/0000-0003-4973-8076

2 Estudiante de Licenciatura en Matemáticas. Departamento de matemáticas y estadística, Universidad de Nariño. Correo: yilbernc98@udenar.edu.co. Orcid: https:// orcid.org/0000-0003-4281-6959 fases: la primera fase se basó en la búsqueda e identificación de situaciones problema en la literatura internacional y nacional, guiados en esta, la recolección del material empírico se centró en artículos de revistas científicas publicados entre 1995 y 2019 . En la segunda fase se procedió a sistematizar las situaciones problema encontradas, y luego separando y describiendo las formas de resolución de dichas situaciones problema en soluciones extraescolares y soluciones escolares. Entre los resultados se puede destacar las habilidades matemáticas que han desarrollado las personas a partir de su experiencia y las actividades encontradas en aritmética y geometría donde llama la atención los diferentes algoritmos mentales diseñados 
por personas no escolarizadas para la solución de un problema.

PALABRAS CLAVE: Etnomatemática; Situaciones problema; Practicas Matemáticas; Matemáticas escolares; Matemáticas extraescolares.

\section{ABSTRACT}

This study aims to describe ways of solving problem situations, from school mathematics and extracurricular mathematics. The problem is framed in the research field of Ethnomathematics, after conducting a review of research in national and international spheres that allude to tensions between academic and extracurricular mathematics. A qualitative and interpretive methodology was used that included two phases: the first phase was based on the search and identification of problem situations in the international and national literature, guided by it, the collection of empirical material focused on articles from scientific journals published between 1995 and 2019. In the second phase, the problem situations encountered were systematized, and then separating and describing the ways of solving these problem situations into extracurricular solutions and school solutions. Among the results, we can highlight the mathematical skills that people have developed from their experience and the activities found in arithmetic and geometry where the different mental algorithms designed by nonschooled people for the solution of a problem are striking.

KEYWORDS: Ethnomathematics; Problem situations; Math Practices; School mathematics; Extracurricular mathematics.

\section{INTRODUCCIÓN}

Actualmente, un aspecto que genera estudios e investigaciones enelcampodelaEtnomatemática son los procesos de generación, difusión e institucionalización de prácticas matemáticas de diferentes grupos culturales (D'Ambrosio, 2008). Una de estas prácticas matemáticas son los algoritmos extraescolares empleados por distintos grupos sociales y culturales en el momento de solucionar una situación problema.

Para profundizar más sobre la solución de situaciones en prácticas extracurriculares, es importante indagar en el campo de investigación de la Etnomatemática. Este concepto fue creado por D'Ambrosio (1985) quien era matemático y educador brasileño, que usó el término para referirse a: "Los procesos matemáticos, símbolos, jergas, mitologías, modelos de razonamientos, etc., practicados por grupos culturales identificados, inclusive clases profesionales". Por otro lado, Casey, citado por (Albertí, 2007) declara que:

"La Etnomatemática es el estudio
que toma en consideración las
culturas en las que surge la
matemática, dado que algunas
actividades humanas requieren
de matemáticas, actividades
tales como: la arquitectura, el
tejido, la costura, la agricultura,
la ornamentación, las prácticas
religiosas y espirituales, etc."
(p.104)

Estas apreciaciones fomentan el respeto por la diversidad cultural, además permiten estudiar los diferentes pensamientos matemáticos que encontramos a diario en la vida profesional, familiar, política o social, de ahí que, la Etnomatemática es una fuente rica para explorar las soluciones y algoritmos extraescolares empleados en grupos identificables.

En la literatura existen investigaciones que han mostrado interés en los desarrollos $\mathrm{y}$ pensamientos matemáticos en contextos extraescolares, Algunos ejemplos son los trabajos de (Albertí, 2007; Ávila, 1993; Carraher 
et al., 1999; Reed \& Lave, 1981). Sin embargo, luego de realizar una revisión de investigaciones en ámbitos nacionales (Aroca, 2010; BlancoÁlvarez, 2011; Charry et al., 2020; Jaramillo, 2011) e internacionales como (Knijnik \& Meregalli, 2012; Oliveras, 1996, 2005, 2006; Oliveras \& Gavarrete, 2012; Santillán \& Zachman, 2009), se encontró que en dichas investigaciones los autores identificaron algunas tensiones entre las matemáticas escolares y extraescolares: por un lado, el reconocimiento como matemáticas, a prácticas extraescolares genera tensión entre el saber escolar y el saber cotidiano, puesto que el primero se considera científico y el segundo no. Por otro lado, demuestran que las investigaciones se han limitado en reconocer las prácticas extraescolares y los conocimientos que emergen de estás, solo como elementos motivadores en su aplicación en el aula.

En este sentido Jaramillo (2011) explícita la dicotomía entre los saberes considerados no académicos y aquellos validados como académicos

"Los saberes escolares reconocidos y legitimados por la academia se superponen sobre los saberes cotidianos reconocidos y legitimados desde y por las prácticas sociales. En estas relaciones de poder establecidas entre estos dos saberes, las estructuras curriculares, además de legitimar únicamente los saberes escolares, impiden la inclusión de los saberes cotidianos que se construyen fuera de la escuela y en dialéctica con las prácticas sociales..." (p.17)

Así mismo Monteiro (2005) declara que "cuando los saberes escolares desconocen o deslegitiman otra forma de conocimientos y de saberes, se genera un modo de exclusión social, pues esto conlleva a la deslegitimación de las prácticas sociales que dan sustento a dichos saberes."
Algunos investigadores como (Cadeia et al., 2010; Carraher et al., 1999; Lave, 1991; Vilela, 2007), resaltan en sus trabajos la existencia de importantes diferencias entre los saberes extraescolares y los escolares. Por un lado, las matemáticas extraescolares se caracterizan por la oralidad y por el uso de estrategias que involucran procedimientos heurísticos $\mathrm{y}$, por otro, las matemáticas presentes en entornos académicos se caracterizan por la utilización del cálculo escrito y algorítmico, que presupone una superioridad de éstas sobre las matemáticas cotidianas. Sin embargo, dentro de contextos académicos se ha evidenciado que los estudiantes no tienen un interés particular en la solución de un problema matemático, desinterés que no sucede dentro de contextos cotidianos, por ejemplo: estudiantes que usan las matemáticas en la vida diaria, vendiendo productos o calculando y repartiendo ganancias, etc. son capaces de hacer cuentas mentalmente de manera tan rápida mientras tardan tanto haciendo la misma práctica con lápiz y papel.

Por tal razón se hace necesario el estudio de los conocimientos matemáticos extraescolares, y bajo el interrogante ¿Cómo se soluciona una situación problema desde las prácticas matemáticas escolares y las practicas matemáticas extraescolares? se quiere reconocer todos los conocimientos que hacen que el saber matemático se amplié, y explorar las posibilidades de establecer vínculos didácticos entre esos conocimientos y los escolares. Ahora bien, desde la Etnomatemática, trabajos como los de (Blanco-Álvarez, 2011; Blanco-Álvarez et al., 2014; Carraher et al., 1999; D'Ambrosio, 2005), y a (Bishop, 1999) con su planteamiento que existen actividades relacionadas con el entorno y cultura matemática (contar, localizar, medir, diseñar, jugar y explicar) nos permitieron lograr el objetivo de estudio ya que estos, muestran que existe un conocimiento que se ha desarrollado fuera del aula, sustentado en la idea de que las matemáticas se pueden dar en 
diferentes estados y momentos y por lo general no necesita de un espacio unificado como laboratorio para darse, formase y aplicarse.

El trabajo tiene como núcleo, las situaciones problema y el estudio los distintos caminos para su solución. A lo largo de este documento se presentarán algunas situaciones problema documentadas en sus respectivos contextos. Se analiza cada una de las situaciones, describiendo los aspectos más generales y relevantes que atañen a las mismas y se especificara el problema a resolver y dos formas de solución una escolar y otra extraescolar.

\section{METODOLOGÍA}

Esta investigación es de tipo documental que según Gil (1999) hace uso de los materiales publicados que no han recibido un tratamiento analítico, o que todavía pueden nuevamente ser estudiados de acuerdo con los objetivos de búsqueda, desarrollada con metodología cualitativa e interpretativa.(Lecanda \& Garrido, 2002)

\section{$2.1 \quad$ Tipos de materiales y métodos de Búsqueda}

La recolección del material empírico se centró en artículos de revistas científicas publicados entre 1995 y 2019. A partir del objetivo de estudio se dio entrada de las palabras claves en bases de datos como Scopus, Springer, Redalyc, Scielo y Google Scholar, obteniendo estudios destacados y relevantes en el área de interés.

De los artículos encontrados se escogieron solo artículos que contenían situaciones problema y en su solución extraescolar se evidencia la utilización de algoritmos mentales, seleccionando así, solo 7 artículos.

\subsection{Sistematización de la información}

Luego de identificar las situaciones problema, se procede a sistematizarlas siguiendo la estructura de la Tabla 1:

\begin{tabular}{|c|c|}
\hline Pasos & Descripción \\
\hline $\begin{array}{l}\text { Análisis del artículo } \\
\text { encontrado }\end{array}$ & $\begin{array}{l}\text { De forma resumida se contextualiza algunas características } \\
\text { del artículo como: el autor, año, título y objetivo de la } \\
\text { investigación. }\end{array}$ \\
\hline $\begin{array}{l}\text { Descripción de la } \\
\text { situación problema }\end{array}$ & $\begin{array}{l}\text { Siguiendo las consideraciones de Alberti (2007) sobre } \\
\text { practica matemática, en este espacio se detalla sobre los } \\
\text { autores (persona que realiza la practica), la tecnología y el } \\
\text { entorno donde se plantea la situación problema. }\end{array}$ \\
\hline Situación Problema & $\begin{array}{l}\text { Se plantea la situación problema a resolver, dando visibilidad } \\
\text { a los procedimientos y el objetivo para realizar la práctica. }\end{array}$ \\
\hline $\begin{array}{l}\text { S o I u c i o n e s } \\
\text { escolares } \quad \text { y } \\
\text { extraescolares }\end{array}$ & $\begin{array}{l}\text { Se describen los procedimientos utilizados para la solución de } \\
\text { la situación problema, separando en soluciones escolares y } \\
\text { extraescolares. }\end{array}$ \\
\hline
\end{tabular}

Tabla 1: Forma de sistematización de las situaciones encontradas encontrada. 
Para el paso de soluciones escolares y extraescolares se presentan en primer lugar, las respuestas textuales entregadas por los entrevistados o las notas de campo tomadas por los investigadores y en algunos casos se complementan con el algoritmo subyacente a las mismas. Los artículos que solo tenían la solución extraescolar se complementaron por los investigadores, presentando así la solución escolar. Finalmente se realiza una descripción de los procedimientos utilizados en los dos contextos.
Debe quedar claro para el lector que las situaciones problema en los artículos no se encuentran estructurados con los pasos de la Tabla 1. Esta es una estructura creada por nosotros como investigadores para realizar el análisis y la descripción de dichas situaciones problema.

\section{ANÁLISIS Y RESULTADOS}

\subsection{Análisis y descripción de soluciones de situaciones problema}

Las situaciones problema se encontraron en los siguientes artículos, sintetizados en la Tabla 2.

\begin{tabular}{|l|l|l|l|}
\hline No. & \multicolumn{1}{|c|}{ (Autor, Año) } & \multicolumn{1}{|c|}{ Título del artículo } & (Vol., No., pp.) \\
\hline 1. & (Valiente, 1995) & $\begin{array}{l}\text { Análisis de cuatro algoritmos operatorios obtenidos } \\
\text { en la investigación de campo con adultos } \\
\text { analfabetas. }\end{array}$ & $\begin{array}{l}\text { Vol.7, No.2, pp. } \\
64-72\end{array}$ \\
\hline 2. & (Amendola, 2018) & $\begin{array}{l}\text { Una comunidad gitana: el conocimiento matemático } \\
\text { puesto en juego para la resolución de problemas } \\
\text { cotidianos }\end{array}$ & $\begin{array}{l}\text { Vol.11, No.3, pp. } \\
37-54\end{array}$ \\
\hline 3. & $\begin{array}{l}\text { (Naresh } \\
\text { Chahine, 2013) }\end{array}$ & $\begin{array}{l}\text { Mapping the Cognitive Competencies of Street } \\
\text { Vendors and Bus Conductors: A Cross-Cultural } \\
\text { Study of Workplace Mathematics }\end{array}$ & $\begin{array}{l}\text { Vol.6, No.3, pp. } \\
7-35\end{array}$ \\
\hline 4. & $\begin{array}{l}\text { (García García et } \\
\text { al., 2014) }\end{array}$ & $\begin{array}{l}\text { La resolución de problemas en un contexto Nuu } \\
\text { Savi: un estudio de casos con niños de sexto grado } \\
\text { de primaria }\end{array}$ & $\begin{array}{l}\text { Vol.26, No.1, pp. } \\
127-152\end{array}$ \\
\hline 5. & $\begin{array}{l}\text { (Schwantes et al.,, } \\
\text { Matemáticas en el lugar de trabajo de los albañiles }\end{array}$ & $\begin{array}{l}\text { Vol.13, No.7, pp. } \\
46-66\end{array}$ \\
\hline 6. & $\begin{array}{l}\text { (Graças } \\
\text { Marinho, 2015) }\end{array}$ & $\begin{array}{l}\text { Explorando a matemática na construção de casas } \\
\text { de alvenarias }\end{array}$ & $\begin{array}{l}\text { Vol.8, No.1, pp. } \\
29-49\end{array}$ \\
\hline 7. & $\begin{array}{l}\text { (Fioriti, 2002) } \\
\text { Conocimiento geométrico de los obreros de } \\
\text { la construcción: conocimiento situado versus } \\
\text { conocimiento escolar }\end{array}$ & $\begin{array}{l}\text { Vol.14, No.1, pp. } \\
120-129\end{array}$ \\
\hline
\end{tabular}

Tabla 2: Artículos seleccionados para el estudio de situaciones problema.

El material empírico seleccionado para el análisis es presentado en la Tabla 2 donde, los artículos 1, 4 y 7 pertenecen a la Revista de Educación Matemática, Los artículos 2, 3 y 6 hacen parte de la Revista Latinoamericana de Etnomatemática. $\mathrm{Y}$ por último el artículo 5 pertenece a la Revista Científica Multidisciplinar Núcleo do Conhecimento. Realizamos el análisis y descripción de las soluciones escolares y extraescolares, de nueve situaciones problema encontradas en los artículos. Los resultados se muestran a continuación. 


\subsubsection{Situaciones problema 1, 2 y 3.}

En el artículo "Análisis de cuatro algoritmos operatorios obtenidos en la investigación de campo con adultos analfabetas", Valiente (1995) investiga los procedimientos realizados por personas no escolarizadas, en la resolución de problemas que involucran las cuatro operaciones aritméticas elementales, a partir de problemas sencillos.

Descripción de las situaciones: Se plantean tres situaciones problema a un grupo de adultos no alfabetizados que utilizan cálculos en ventas de diferentes objetos y servicios. La situación problema 1 fue planteada a una señora de 79 años de edad dedicada a la venta de objetos de jarcería (elementos elaborados en fibra). La situación problema 2 a Inocente de 67 años de edad cuyo nivel de formación solo va hasta tercero de primaria, la situación problema 3 fue abordada a Inés de 63 años de edad cuyo nivel de formación solo va hasta tercero de primaria.

Situación problema 1: "Usted tiene $\$ 18$ y le dan $\$ 23$ (pesos) ¿Cuántos tiene ahora? ¿Qué hizo para llegar al resultado?"

Solución Extraescolar: Ante el planteamiento ¿Cuántos tiene ahora? La vendedora responde "41". Y para el interrogante ¿Qué hizo para llegar al resultado? La vendedora respondió: "18 más 3,21 y 20,41 "

Mentalmente la vendedora descompone el número mayor en 2 decenas y 3 unidades. $\mathrm{Al}$ número menor le suma las 3 unidades: 18 más 3 igual a 21 . A 21 le suma las 2 decenas: 21 más 20 igual a 41.

Solución escolar - Algoritmo escolar: Para la solución escolar de la situación problema 1 se debe emplear el algoritmo para sumar números o cantidades. Se comienza a sumar de derecha a izquierda. Sumar 8 unidades con 3 unidades, tenemos una decena y una unidad. Se coloca la unidad debajo de la primera columna y la decena pasa a la segunda columna. Se suma 1 decena con 1 decena y 2 decenas, tenemos 4 decenas. Se coloca las 4 decenas debajo de la segunda columna. El resultado es 41 .

Situación problema 2: "Usted cobra $\$ 72$ por un servicio y le pagan con un billete de $\$ 100$. Explique cómo daría el cambio"

Solución Extraescolar: el investigador pide a Inocente que explique cómo daría el cambio y ella responde "70 y 30 son 100, menos 2 son los 28 ".

Inocente toma el dato menor 72 e implícitamente le resta 2 unidades: 70 menos 2 igual 70 . Donde 70 representa las decenas del dato menor. A las decenas del dato menor le suma 30 , donde 30 es el complemento del dato mayor 100 ya que 70 más 30 es igual a 100. Al complemento le resta las 2 unidades del dato menor: 30 menos 2 igual a 28 . El resultado es igual a 28 pesos.

Solución escolar - Algoritmo escolar: para la solución escolar empleamos el algoritmo para restar números o cantidades. Así pues, se comienza a restar de derecha a izquierda. En la columna de las unidades: 10 menos 2 igual 8 unidades. En la columna de las decenas: 9 menos 7 igual a 2 unidades. La columna de las centenas se convierte en cero. El resultado es igual a 72 pesos.

Situación problema 3: "Si el kilogramo de un producto cuesta \$3.300. ¿Cuánto costará medio kilo?"

Solución extraescolar: Ante el planteamiento del investigador ¿Cuánto costará medio kilo? Inés responde: " 3.000 entre dos es igual a 1.500, 300 entre dos es igual a 150, 1.500 más 150 es igual a $1.650 "$

Inés descompone a 3.300 en 3 unidades de mil y 3 centenas, luego divide las 3 unidades de mil entre 2 y obtiene un primer valor parcial 1500. Divide las 3 centenas entre 2 y obtiene 
un segundo valor parcial, suma los dos valores parciales: 1.500 más 150 y el resultado es igual a $\$ 1.650$.

Solución escolar - Algoritmo escolar: Para la solución escolar de la situación problema 3 se debe emplear el algoritmo para dividir números o cantidades. Para este caso el dividendo será 3.300 porque es la cantidad que queremos dividir y 2 es el divisor. Para hallar el cociente tomamos la primera cifra del dividendo, si esta cifra es más pequeña que el divisor entonces se debe tomar otra cifra del dividendo. En este caso 3 es mayor que 2 por lo tanto se toma una sola cifra del dividendo. Se busca un número que al multiplicar por 2 dé como resultado el dividendo 3 , si no lo hay se busca el resultado menor próximo, para este caso se multiplica 2 por 1. El resultado de la multiplicación se resta al dividendo: a 3 le restamos 2 y tenemos 1 . Se baja la siguiente cifra del dividendo y se realiza de nuevo el paso 3 . El resultado es igual a $\$ 1.650$

\subsubsection{Situación problema 4}

En el artículo "Una comunidad gitana: el conocimiento matemático puesto en juego para la resolución de problemas cotidianos", Amendola (2018) analiza los conocimientos matemáticos que una comunidad gitana pone en juego a la hora de resolver problemas que involucran actividades cotidianas.

Descripción de la situación: Se plantean diferentes situaciones problema a miembros de una comunidad gitana argentina, cuya actividad laboral es, en su mayoría, el comercio de diferentes productos en plazas y su nivel de formación académica es baja o inexistente en algunos casos. Se toma una situación como ejemplo para realizar el análisis y descripción.
Situación problema 4: "Si compro 3 cosas que cuestan $\$ 1,25$ cada una y tengo que pagar con un billete de $\$ 5$ (pesos argentinos). ¿Cuánto me dan de vuelto?"

Solución extraescolar: Un miembro de la comunidad responde: "Son tres setenta y cinco. [...] Primero saqué la de 1 peso y después saqué la de $25 \ldots 1,2,3,75$ y me faltan 25 para un peso y 1 peso son cinco".

Analizando la respuesta de la comerciante, cada objeto cuesta $1,25 \$$. Primero suma 1 peso tres veces, que corresponde a la parte entera del precio individual de cada objeto. Luego suma 25 tres veces que es igual a 75 y representa la parte decimal del precio individual de cada objeto. Luego suma 0,25 al 0,75 y completa un peso, de esta forma completa en total 4 pesos. Luego a 4 pesos le suma 1 peso y completa los 5 pesos. Finalmente, suma los complementos 0,25 y 1 peso, obteniendo 1,25 , que corresponde al valor del vuelto.

Solución escolar - Algoritmo escolar: Para la solución escolar empleamos la combinación de algoritmos para multiplicar y restar números o cantidades decimales.

Primero se multiplica 1,25 por el número de cosas, considerando los dos factores como números enteros se multiplica 3 por 5 igual a 15 se escribe el 5 en la primera columna y 1 pasa a sumar a la segunda columna. Se multiplica 3 por 2 igual a 6, así en la segunda columna se suma 6 más 1 igual a 7 . En la tercera columna se multiplica 3 por 1 igual a 3 . Posteriormente se cuentan cuantos dígitos son decimales en los factores, en este caso 1,25 tiene dos decimales por lo tanto en el resultado se cuentan dos espacios de derecha a izquierda y se coloca la coma en dicha posición, así, el valor resultante es igual a $\$ 3,75$. Finalmente se resta $\$ 3,75$ a 5 y el vuelto es igual a $\$ 1.25$. 


\subsubsection{Situación problema 5}

En el artículo "Mapeo de las competencias cognitivas de los vendedores ambulantes y los conductores de autobuses: Un estudio transcultural del lugar de trabajo de Matemáticas", Naresh \& Chahine (2013) investigan las ideas matemáticas que surgen de dos lugares de trabajo, la venta ambulante y la conducción de autobús. La finalidad de su investigación fue definir una dirección que describa posibles estructuras matemáticas implícitas en la conducción de autobuses y actividades de venta ambulante para extraer un modelo conceptual que podría explicar la naturaleza del conocimiento matemático de los practicantes y su conexión con las matemáticas formales.

Descripción de las situaciones: Se plantea una situación problema a un vendedor ambulante. Las personas escogidas en esta investigación al menos han completado su educación secundaria y están empleados por el MTC, una agencia gubernamental del Líbano. Durante las entrevistas informales y semiestructuradas, se utilizaron un método de recuerdo estimulado para ayudar a los participantes a reflexionar sobre sus actos de cálculo mental.

Situación problema 5: Masri vende cebollas, 750L.L el 1 kilo: El investigador le plantea "Quiero 3 kilos de cebollas, ¿cuánto te debo?" (L.L libra libanesa)

Solución Extraescolar: El investigador observa que Masri realiza mentalmente los cálculos y responde: " 2 kilos por 750 L.L más 750L.L que da 1.500L.L, y otro 1 kilo por 750L.L ...luego 2.250L.L"

Esta situación puede ser considerada como un problema de multiplicación, precisamente
750 por 3. Sin embargo, Masri no utilizó este algoritmo más bien, resolvió el problema por medio de adiciones repetidas de la siguiente manera:

$$
\begin{gathered}
1 \text { kilo--- } \\
1+1=2 \text { kilos---------750 + } 750 \text { = 1.500 L.L } \\
2+1=3 \text { kilos---------1.500 + } 750=2.250 \mathrm{LL}
\end{gathered}
$$

Solución escolar: Una forma escolar para la solución de esta situación es establecer una relación entre el peso y el precio, es decir un mapeo de (una función): a cada peso le corresponde un precio bien definido, luego, se aplica la propiedad descrita por Vergnaud (1988), como sigue:

$$
g(X+Y)=g(X)+g(Y)
$$

Definida como la propiedad isomorfa de la suma o la propiedad lineal de la función $g$. Las variables peso y precio son independientes y mantienen sus valores proporcionales. Al vender a un precio $A$, el vendedor era consciente de que un aumento en el número de kilos corresponde a un aumento proporcional en el precio. Representando la solución anterior explícitamente: $g=\operatorname{costo}(x)$, donde $x$ es el número de kilos, como costo ( 1 kilo $)=750$ L.L.

entonces: Por la propiedad lineal:

$$
\begin{gathered}
\operatorname{costo}(3 \text { kilos })=\operatorname{costo~}(1 \text { kilo }+1 \text { kilo }+1 \text { kilo })= \\
\text { costo }(1 \text { kilo })+\operatorname{costo~}(1 \text { kilo })+\operatorname{costo}(1 \text { kilo })= \\
3 \text { costo }(1 \text { kilo })
\end{gathered}
$$

Remplazando 3 x 750 L. L $=2250$ L.L

\subsubsection{Situación problema 6}

Este artículo "La resolución de problemas en un contexto Ñuu Savi: un estudio de casos con niños de sexto grado de primaria", García, Navarro \& Rodríguez (2014) presentan resultados de una investigación que pretendió mostrar algunas estrategias identificadas en las producciones de 
alumnos de sexto grado al resolver problemas aritméticos: formales y prácticos.

Descripción de la situación: Se plantea una situación problema a un niño Tee Savi perteneciente a la población Ñuu Savi (mixteca) del estado de Guerrero, México, donde se utiliza la estrategia "lista los casos posibles"

Situación problema 6: "En una nevería se venden los siguientes sabores: fresa, vainilla, limón y chocolate, encuentra todas las formas diferentes de servir un helado de dos sabores"

Solución Extraescolar:

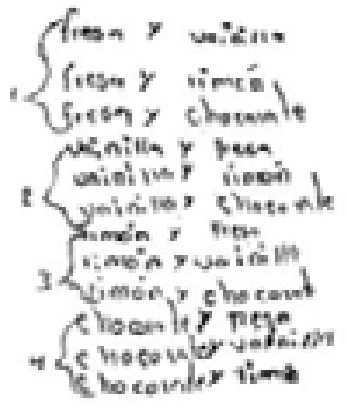

Figura 1: Resolución de un problema donde se utiliza la estrategia "lista los casos posibles"

(García García et al., 2014, p. 144)

Como se observa en la Figura 1, el niño construyó una lista de las posibles formas de servir un helado de dos sabores; sin embargo, le faltó discriminar aquellos casos donde la combinación era la misma, por ejemplo: limón y chocolate que es la misma que chocolate y limón. En la respuesta del niño, consigue mostrar las seis combinaciones que se derivan de las exigencias dadas en el problema y que es la solución de este. En general, la estrategia es muy útil, solo falta tener cuidado con las repeticiones.

\section{Solución extraescolar - Algoritmo} extraescolar: Reemplazamos los datos en la fórmula y obtenemos la respuesta:

$$
C(n, x)=\left(\begin{array}{l}
n \\
x
\end{array}\right)=\frac{n !}{x !(n-x) !}
$$

Para obtener las diferentes formas de servir un helado, matemáticamente se puede hacer uso de una combinación sin repetición definida como a los diferentes conjuntos que se pueden formar con $n$ elementos, seleccionados de $x$ en $x$. Cada conjunto se debe diferenciar del anterior en al menos uno de sus elementos (el orden no importa) y estos no se pueden repetir.

$$
\mathrm{C}(4,2)=\left(\begin{array}{l}
4 \\
2
\end{array}\right)=\frac{4 !}{2 !(4-2) !}=6
$$

\subsubsection{Situación problema 7}

En el artículo "Matemáticas en el lugar de trabajo de los albañiles" Moreira \& Pardal (2012) presentan las prácticas profesionales de los albañiles, que están relacionadas con las matemáticas para contribuir al área de la educación matemática de adultos y ampliar el conocimiento sobre cómo se utilizan las matemáticas en esta labor.

Descripción de la situación: Se propone una situación problema a Don Antonio, un albañil durante 46 años, con estudios hasta el tercer grado, respecto al cálculo del ángulo de inclinación del techo.

Situación problema 7: "Si el techo tiene $3 \mathrm{~m}$ en horizontal y 1,5 m en vertical, ¿cuál será la inclinación?

Solución Extraescolar: Don Antonio responde: "Conozco la altura de la cresta de la raíz (1,5 m) y el valor de la línea horizontal (3 m); por lo tanto, divido la cresta de la raíz por la línea horizontal (1,5 m: $3 \mathrm{~m})$. El resultado de la división es 0,5. Luego lo multiplico por 100 para obtener el valor del porcentaje, y es $50 \%$ “

Lo primero que hace Don Antonio es tener en cuenta los valores: línea vertical $(1,5 \mathrm{~m})$ y la línea horizontal $(3 \mathrm{~m})$. Divide el dato de la línea vertical $(1,5)$ sobre el dato de la línea horizontal $(3 \mathrm{~m})$. Obtiene como resultado $\frac{1,5 m}{3 m}=0,5$. 
El resultado obtenido lo multiplica por 100 para obtener el porcentaje

\section{Solución escolar- Algoritmo extraescolar:} Resolvemos la situación mediante el uso de teoremas y definiciones:

$$
\begin{gathered}
\mathrm{h}^{2}=\mathrm{A}^{2}+\mathrm{B}^{2}=\mathrm{h}^{2}=(1,5)^{2}+(3)^{2} \\
\mathrm{~h}=\sqrt{11,25} \\
\frac{\mathrm{a}}{\sin \mathrm{A}}=\frac{\mathrm{c}}{\sin \mathrm{C}} \rightarrow \frac{1,5}{\sin \mathrm{A}} \\
\frac{\sqrt{11,25}}{1} \rightarrow \sin \mathrm{A}=\frac{1,5}{\sqrt{11,25}} \\
\mathrm{~A}=\sin ^{-1} \frac{1,5}{\sqrt{11,25}} \rightarrow \mathrm{A} \approx 0,4472 \ldots \\
0,4472 \ldots * 1,75=51,65 \ldots \%
\end{gathered}
$$

Primero, para determinar la longitud de la hipotenusa (techo) se utiliza el teorema de Pitágoras. Como se tiene los valores $(1,5 \mathrm{~m})$ y (3m). Se reemplaza y se obtiene $h=\sqrt{11,25}$.

Mediante el teorema de senos para determinar el ángulo de inclinación. Se reemplaza los valores y se obtiene $A \approx 0,4472$.

Finalmente, mediante una regla de tres buscamos la equivalencia en porcentaje y se obtiene $51,65 \ldots \%$

\subsubsection{Situación problema 8}

En el artículo "Explorando las matemáticas en la construcción de casas de mampostería", Graças Castro \& Marinho Fonseca (2015) describen las prácticas matemáticas desarrolladas en las actividades que realizan los albañiles en la construcción de casas utilizando una investigación de tipo cualitativo a través de entrevistas no estructuradas con preguntas abiertas y observación directa para comprender las ideas y razonamientos matemáticos existentes en este proceso.
Descripción de la situación: Se plantea situación problema a un albañil, cuyo objetivo es determinar la cantidad de ladrillos que debe utilizar para cubrir un área determinada, la investigación fue desarrollada en del municipio de Urucará Brasil.

Situación problema 8: "Calcular la cantidad de ladrillos necesarios para el levantamiento de cada metro cuadrado de muro. Si cada ladrillo tiene dimensiones de $19 \mathrm{~cm}$ x $19 \mathrm{~cm} \times 9 \mathrm{~cm}$ "

Solución Extraescolar: Para calcular la cantidad de ladrillos necesarios para la construcción es un problema de área de superficies rectangulares. La mayoría de los albañiles ya conocen la cantidad de ladrillos por metro cuadrado, producto de su experiencia. La cantidad requerida de ladrillos varía de 25 a 28 ladrillos por metro cuadrado, descontando la masa entre los ladrillos.

El albañil no calcula utilizando fórmulas matemáticas abstractas aprendidas en un entorno escolar, simplemente ya sabe por experiencia la cantidad necesaria para un metro cuadrado y por lo tanto hace la estimación propuesta en el trabajo.

\section{Solución escolar- Algoritmo escolar:} Calculamos por medio de una fórmula matemática para calcular la cantidad de ladrillos en un metro cuadrado

$$
\text { Número de ladrillos }=\frac{1}{\text { ancho } \times \text { largo }}
$$

Ladrillo (considerando dimensiones) $A=19 \mathrm{~cm}$ o $0,19 \mathrm{~m}$ y $\mathrm{L}=19 \mathrm{~cm}$ o $0,19 \mathrm{~m}$

$$
\begin{aligned}
& \mathrm{T}=\frac{1}{0,19 \times 0,19}=\frac{1}{0.0361} \\
& \mathrm{~T}=27,7 \text { ladrillos por } \mathrm{m}^{2}
\end{aligned}
$$

Este cálculo se puede hacer con la ayuda de una fórmula matemática, multiplicando su largo por su ancho y dividiendo $1 \mathrm{~m}^{2}$ por el producto obtenido De esta forma, se calcula cuántos ladrillos serán necesarios para el levantamiento de cada metro cuadrado de muro. 


\subsubsection{Situación problema 9}

En el artículo "Conocimiento geométrico de los obreros de la construcción: conocimiento situado versus conocimiento escolar", Fioriti (2002) investiga los conocimientos en la realización de diferentes prácticas cotidianas. En particular del conocimiento matemático en el trabajo que realizan los obreros de la construcción.

Descripción de la situación: Se plantea una situación problema a un obrero, el propósito es identificar los procedimientos para controlar la escuadra, es decir el ángulo recto en la intersección de dos paredes o en las aberturas correspondientes a puertas y ventanas.

Situación problema 9: “¿Cómo se obtiene un ángulo recto en la construcción de las paredes de una habitación o los lados de una ventana? (escuadra se refiere ángulos rectos $\left.90^{\circ}\right)$ "

Algoritmo Extraescolar: El obrero responde: "Por ejemplo, la escuadra nosotros la tomamos, en una parte nos da los 60 , en la otra parte nos damos 80 y ahí vemos, si nos da el metro es porque está en escuadra, ... pero si nos pasamos un poquito ya significa que está fuera de escuadra ..."

Es necesario señalar que el procedimiento realizado por el obrero, son transmitidas verbalmente de unos a otros provocando que este sea reconocido como un saber intuido. Utiliza una forma de razonamiento (recíproca del teorema de Pitágoras). En general los obreros miden $0,60 \mathrm{~m}$ y $0,80 \mathrm{~m}$ en los catetos y a continuación Im para la hipotenusa. Si en un triángulo $A B C, A B=0,80 \mathrm{~m}, A C=0,60 \mathrm{~m}$ y $B C$ $=1 \mathrm{~m}$ entonces el triángulo $A B C$ es rectángulo en $\mathrm{A}$.

Solución Escolar - Algoritmo escolar: Para la solución escolar se utiliza el teorema de Pitágoras que establece que, en todo triángulo rectángulo, la longitud de la hipotenusa es igual a la raíz cuadrada de la suma del área de los cuadrados de las respectivas longitudes de los catetos.

Datos: $a=0,80 \mathrm{~m}$ y $b=0,60 \mathrm{~m}$ tenemos:

$$
h^{2}=(0,8 m)^{2}+(0,6 m)^{2}=1 m
$$

Dadas las medidas de los catetos 1 y 2 del triángulo. Dado que el triángulo tiene un ángulo recto, entonces puedes calcular la hipotenusa por medio de $h^{2}=a^{2}+b^{2}$.

En este caso a y $b$ son los catetos

\section{CONCLUSIONES Y DISCUSION}

De la presente investigación, se pudo evidenciar que, a lo largo de la historia las personas y las culturas han venido construyendo conocimientos y saberes que tienen relación directa con las matemáticas, pues hoy en día encontramos registros y actividades en los cuales se evidencia la existencia y necesidad de un conocimiento matemático. Con el paso del tiempo, estos conocimientos se han sistematizado y registrado en lo que llamamos las matemáticas, a este respecto, se parte de entender a la matemática como un conocimiento de construcción social y por tal razón se encuentra teñido por distintas gamas que provienen tanto de las ideas filosóficas, sociales, culturales, históricas, religiosas, entre otros tantos aspectos que describen a una sociedad en un lugar y tiempo determinado.

En este marco, se puede concluir que la Etnomatemática estudia los conocimientos matemáticos cotidianos y aquellos que son producidos por diversas culturas, este hecho ayuda al reconocimiento y revalorización de los saberes y las prácticas matemáticas de las comunidades y de las diferentes culturas. De lo anterior podemos decir que, las matemáticas extraescolares tienen otras rutas de validación, su validez se comprueba en el día a día, son parte de la vida cotidiana y pueden contribuir al conocimiento científico matemático. 
Hoy en día es muy común mirar en libros o en clases de matemáticas problemas contextualizados, a tal punto que se intenta llevar una especie de subrealidad al aula de clase, con el objetivo de que punto teórico se vuelvan prácticos para que los estudiantes intenten dotar de sentido a los procesos matemáticos, sin embargo, este tipo de prácticas con lleva a confusión en los estudiantes. Pues no solo se trata de tener en cuenta unas supuestas situaciones cotidianas como desarrollar ejemplos con manzanas, peras, etc. La búsqueda, análisis y estudio sobre situaciones problema en prácticas cotidianas donde se hacen presentes las matemáticas, han logrado ampliar nuestra forma de ver las matemáticas que desarrollan en un ambiente no escolar, de tal modo, que vemos un valor de la enseñanza de las matemáticas contextualizada y basada en un aprendizaje de las prácticas de grupos sociales, de esta manera se debería plantear una educación etnomatemática más arraigada con la cotidianidad, donde se quiera dotar de significado social y cultural, partiendo de la lógica que genera cada situación, poniéndose así más al alcance de los estudiantes para que ellos puedan acercarse mucho a las matemáticas formales partiendo de un entorno familiar en el que habitan.

Es así que en la presente investigación se logró describir formas de solución para situaciones problema, y responder el planteamiento de investigación. Por un lado, las situaciones problema 1, 2, 3, 4 y 5 nos permiten evidenciar que no existen cálculos o algoritmos únicos para desarrollar las operaciones aritméticas fundamentales, sino que hay un cálculo flexible y diversidad de procedimientos que permiten llegar a un resultado acertado, estos procedimientos $\mathrm{o}$ algoritmos se desarrollan dependiendo del contexto y la forma en que se presente la situación.
De igual manera la situación problema 6 relacionada con el cálculo de probabilidades o las situaciones 7,8 y 9 relacionadas con pensamientos geométricos y trigonométricos como el teorema de Pitágoras, nos muestras otros caminos de solución y nos permiten explorar las posibilidades de establecer vínculos didácticos entre esos conocimientos y los escolares. De hecho, los algoritmos extraescolares descritos pueden convertirse en caminos válidos para la solución de un problema.

Finalmente se puede concluir que la perspectiva de la Etnomatemática permite que una sugerencia educativa funcione en el aula, alienta a los estudiantes y maestros a desarrollar la creatividad y producir estilos de aprendizaje nuevos y ricos. $Y$ en relación a los algoritmos encontrados y analizados, se puede decir que, la Etnomatemática como línea de investigación, es un programa que ayuda, contribuye y aporta mucho valor a la educación matemática, en este sentido ayuda en gran manera a la enseñanza de las matemáticas, ya que, permite ver a la matemática como una disciplina que se puede lidiar desde contextos de la cotidianidad.

\section{REFERENCIAS BIBLIOGRÁFICAS}

Albertí, M. (2007). Interpretación situada de una Práctica Artesanal. Tesis doctoral. Universidad Autónoma de Barcelona, España.

Amendola, M. (2018). Una comunidad gitana : el conocimiento matemático puesto en juego para la resolución de problemas cotidianos. Revista Latinoamericana de Etnomatemática, 11, 37-54.

Aroca, A. (2010). Una experiencia de formación docente en Etnomatemáticas: estudiantes afrodescendientes del Puerto de Buenaventura, Colombia. Revista Horizontes, 28(June 2010), 87-95. 
Ávila, A. (1993). El saber matemático extraescolar en los libros para la educación de adultos. Educación Matemática, 05(03), 60-77.

Bishop, A. J. (1999). Enculturación matemática: la educación matemática desde una perspectiva cultural. Traducido por G. Sánchez Barberán, Barcelona, Paidós Ibérica.

Blanco-Álvarez, H. (2011). La postura sociocultural de la educación matemática y sus implicaciones en la escuela. Revista Educación y Pedagogía, 23(59), 59-66.

Blanco-Álvarez, H., Higuita, C., \& Oliveras, M. L. (2014). Una mirada a la Etnomatemática y la Educación Matemática en Colombia : caminos recorridos $A$ look to the Ethnomathematics and the Mathematics Education in. Revista Latinoamericana de Etnomatemática, 7(2), 245-269. https:// www.redalyc.org/pdf/2740/274031870016. pdf

Cadeia, C., Palhares, P., \& Sarmento, M. (2010). As crianças ciganas nas feiras e na escolaos seus métodos de cálculo mental. Quadrante, 19(1), 71-92.

Carraher, T., Carraher, D., \& Schliemann, A. (1999). Libro En La Vida Diez Y En La Escuela Cero.

Charry, Ó., Jaramillo, D., \& Tamayo, C. (2020). Currículo [de matemáticas]: una problematización de la dicotomía teoría/ práctica desde un territorio chocoano. Educação \& Realidade, 45(3), 1-23. https://doi.org/10.1590/2175-6236106760

D'Ambrosio, U. (1985). Ethnomathematics and its Place in the History and Pedagogy of Mathematics. For the Learning of Mathematics, 1, 44-48.
D’Ambrosio, U. (2005). La integración de la matemática con las ciencias. Matematicalia: Revista Digital de Divulgación Matemática de La Real Sociedad Matemática Española, 1(1), 6.

D'Ambrosio, U. (2008). Etnomatemática. Entre las tradiciones y la modernidad. (A. Editora (ed.)). Limusa.

Fioriti, G. (2002). Conocimiento geométrico de los obreros de la construcción: conocimiento situado versus conocimiento escolar. Educación Matemática, 14(1), 120-129.

García García, J., Navarro Sandoval, C., \& Rodríquez Vasquez, F. (2014). La resolución de problemas en un contexto Ñuu Savi:1 un estudio de casos con niños de sexto grado de primaria. Educación Matemática, 26(1), 127-152.

Gil, A. C. (1999). Métodos e técnicas de pesquisa social (Atlas (ed.); 5th ed.).

Graças Castro, A., \& Marinho Fonseca, J. (2015). Explorando a matemática na construção de casas de alvenarias. Revista Latinoamericana de Etnomatemática: Perspectivas Socioculturales de La Educación Matemática, 8(1), 29-49.

Jaramillo, D. (2011). La educación matemática en una perspectiva sociocultural: tensiones, utopías, futuros posibles. Revista Educación y Pedagogía, 23(59), 13-36. http://aprendeenlinea.udea.edu. co/revistas/index.php/revistaeyp/article/ view/8688

Knijnik, G., \& Meregalli, S. J. (2012). Educação matemática em cursos de Pedagogia : um estudo com professores brasileiros dos anos iniciais de escolarização Mathematics 
education in Teacher Education courses : a study with Brazilian teachers of first years of schooling. Revista Latinoamericana de Etnomatemática, 5, 4-20.

Lave, J. (1991). La cognición en la práctica. Paidós.Barcelona.

Lecanda, R., \& Garrido, C. (2002). Introducción a la metodología de investigación cualitativa. Revista de Psicodidáctica, 14, 5-39. http://www.redalyc.org/ html/175/17501402/\%0Ahttp://www. redalyc.org/resumen.oa?id=17501402

Monteiro,A. (2005). Currículo de Matemáticas: reflexões numa perspectiva enomatemática, 7. ${ }^{\circ}$ Encuen- tro de Educación Matemática, Asocolme, Tunja. Encuen- Tro de Educación Matemática, Asocolme, Tunja.

Moreira, D., \& Pardal, E. (2012). Mathematics in Masons' Workplace . Adults Learning Mathematics , 7(1), 32-48. https://search. ebscohost.com/login.aspx?direct=true \&db $=$ eric $\& A N=E J 1068247 \&$ site $=$ ehost-live

Naresh, N., \& Chahine, I. C. (2013). Mapping the Cognitive Competencies of Street Vendors and Bus Conductors: A Cross-Cultural Study of Workplace Mathematics. Revista Latinoamericana de Etnomatemática, 6(3), 7-35.

Oliveras, M. L. (1996). Etnomatemáticas. Formación de profesores e innovación curricular. Comares.

Oliveras, M. L. (2005). Microproyectos para la educación intercultural en Europa. Uno: Revista de Didáctica de Las Matemáticas, España, 38, 70-81.

Oliveras, M. L. (2006). Etnomatemáticas. De la multiculturalidad al mestizaje. Matemáticas e Interculturalidad, 117-149.
Oliveras, M. L., \& Gavarrete, M. E. (2012). Modelo de aplicación de etnomatemáticas en la formación de profesores para contextos indígenas en Costa Rica. RELIME: Revista Latinoamericana de Investigación En Matemática Educativa, 15, 339-372.

Reed, H. J., \& Lave, J. (1981). Arithmetic As a Tool for Investigating Relations Between Culture and Cognition. American Ethnologist, 6(3), 568-582. https://doi. org/10.1525/ae.1979.6.3.02a00100

Santillán, A., \& Zachman, P. (2009). Una experiencia de capacitación en Etnomatemática. Revista Latinoamericana de Etnomatemática: Perspectivas Socioculturales de La Educación Matemática, 2(1), 27-42.

Schwantes, V., Xavier, M. P., Schwantes, E. B., Schwantes, D., Junior, A. C. G., Kracke, E., \& Junior, É. C. (2019). Etnomatemática: Una reflexión sobre las matemáticas utilizadas por los albañiles. Revista Científica Multidisciplinar Núcleo Do Conhecimento, 13(07), 46-66. https://doi.org/10.32749/ nucleodoconhecimento.com.br/educaciones/etnomatematica-una-reflexion

Valiente Barderas, S. (1995). Análisis de cuatro algoritmos operatorios obtenidos en la investigación de campo con adultos analfabetas. Educacion Matemática, 7 (2), 64-72.

Vergnaud, G. (1988). Estructuras multiplicativas. En J. Hiebert y M. Behr (Eds.). Númeroconceptos y Operaciones En Los Grados Intermedios, 46-65.

Vilela, D. S. (2007). Matemática nos usos e jogos de linguagem: Ampliando concepções na Educação Matemática. Unicamp, Campinas, Brasil. 